
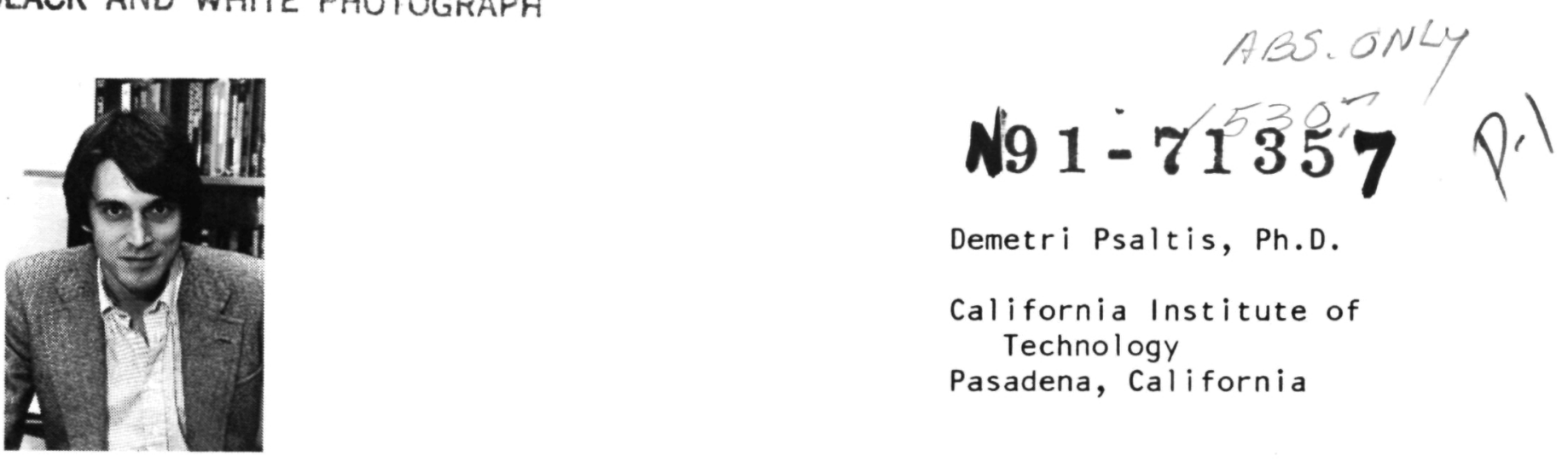

Demetri Psaltis, Ph.D.

California Institute of Technology

Pasadena, California

Dr. Psaltis received his B.S. in electrical engineering and economics in 1974 and his M.Sc. and Ph.D. degrees in electrical engineering in 1975 and 1977, respectively, all from Carnegie-Mellon University, Pittsburgh, Pennsylvania. Upon completion of the Ph.D. program, he remained at Carnegie-Mellon as a research associate, and later as a visiting assistant professor, for a period of 3 years. In 1980, Dr. Psaltis joined the faculty of the electrical engineering department at the California Institute of Technology, Pasadena, where he is now an associate professor and consultant to industry. His research interests are in the areas of optical information processing, acoustooptics, image processing, pattern recognition, neural network models of computation, and optical devices. He has written more than 130 technical publications, is a fellow of the Optical Society of America, and is vice president of the International Neural Networks Society.

\title{
OPTICAL NEURAL COMPUTERS
}

\section{Abstract}

A neural computer consists of a large number of simple processing elements (neurons) that are densely interconnected. The information that is needed to solve a particular problem is stored in the strength of the interconnections using a learning procedure. Some of the basic characteristics of such a computer and the class of problems for which it is best suited are discussed. Optics is a technology particularly well suited for implementing neural computers because of the relative ease with which a programmable, massive interconnection network can be optically synthesized. Several experimental demonstrations of optical networks will be described and the ultimate capabilities of optical neural computers will be projected. 